\title{
Concept learning and feature interpretation
}

\author{
THOMAS L. SPALDING \\ University of Iowa, Iowa City, Lowa \\ and \\ BRIAN H. ROSS \\ University of Illinois at Urbana-Champaign, Urbana, Illinois
}

\begin{abstract}
Models of categorization often assume that people classify new instances directly on the basis of the presented, observable features. Recent research, however, has suggested that the coherence of a category may depend in part on more abstract features that can link together observable features that might otherwise seem to have little similarity. Thus, category learning may also involve the determination of the appropriate abstract features that underlie a category and link together the observable features. We show in four experiments that observable features of a category member are often interpreted as congruent with abstract features that are suggested by observable features of other highly available category members. Our discussion focuses on the implications of these findings for future research.
\end{abstract}

Categories provide a basis for much intelligent cognition and action - for prediction, induction, explanation, communication, inference, and problem solving. Members of a category are grouped together because they are alike in some important respects. In some cases, the physical similarity of items seems great (e.g., silver maple trees). In other cases, however, the similarity of category members is at a more abstract level than what their observable features would suggest; such would be the case for the categories environmentalists, healthy people, and pets. Individual members in these categories differ greatly from one another, even with regard to category-related features; however, we are able to understand that they are similar at a more abstract level. For example, all pets provide companionship, but pet birds and pet dogs do so in very different ways and their physical features are very different from each other. This category of very diverse objects is a good example, though, in that it provides a rich source for prediction (e.g., How will Valerie feel if her pet canary dies?), induction, and explanation. The goal of the present article is to examine how abstract features may be activated by the comparisons of instances and then used for interpreting additional instances.

The obvious physical similarities of many natural categories have led to a general belief that concepts have a family-resemblance structure, such that members of the

\footnotetext{
The research and preparation of this paper were partially supported by Grant SBR 97-20304 from the National Science Foundation. Part of the work was conducted at the Beckman Institute for Advanced Science and Technology. We thank Mary Lassaline, Gregory Murphy, Lisa Oakes, and Jodie Plumert for comments on an earlier version of this article. We thank Amanda Lorenz, Bradley McDowell, and Victoria Phillips for their help in testing participants. Correspondence may be addressed to T. L. Spalding, University of Western Ontario, Department of Psychology, Social Science Center, London, ON, N6A 5C2 Canada or to B. H. Ross, University of Illinois, Department of Psychology, $603 \mathrm{E}$. Daniel St., Champaign, IL 61820 (e-mail: thomas-spalding@uiowa.edu).
}

same category share many features and members of different categories usually share few features (see, e.g., Komatsu, 1992; Rosch, 1975, 1978; Rosch \& Mervis, 1975; Smith \& Medin, 1981). Theories of categorization and category representation have generally assumed that features are given (either by the world or by some earlier or lower level processes); but they have not addressed how features are created or discovered (e.g., Hintzman, 1986, 1988; Medin \& Schaffer, 1978; Posner \& Keele, 1968, 1970; see Wisniewski \& Medin, 1994, for a discussion). In this view, the learning of categories amounts to discovering how the given features are distributed among the categories, or, alternatively, inventing a set of categories that captures the important aspects of the distribution of the given features.

Recently, researchers interested in concepts have begun to question the idea that the features of a concept are given. What is considered as a feature must be constrained in some way, such as by our knowledge, our theories about the world, and our previous experience (see, e.g., Goldstone, 1995; Medin, 1989; Murphy, 1993; Murphy \& Medin, 1985; Murphy \& Spalding, 1995; Pevtzow \& Goldstone, 1994; Schyns \& Murphy, 1994; Schyns \& Rodet, 1997; Thibaut \& Schyns, 1995; see Schyns, Goldstone, \& Thibaut, 1998, for a summary). Given that category features are not fixed, we must account for how a particular set of category features arises. One early suggestion (see, e.g., Murphy \& Medin, 1985) was that a person selects the relevant features on the basis of the person's background knowledge or naive theories about the domain, and then simply learns about the empirical regularities among the selected features. Although recent research has suggested that this simple explanation is not sufficient to explain all the effects that background knowledge has on how participants learn about empirical regularities in the features of presented instances (see, e.g., Spalding \& Ford, 1998; Spalding \& Murphy, 1999; Spalding \& Phillips, 1998; 
Wisniewski, 1995), background knowledge may be very important in how people identify the features of a category. In general, it seems likely that what is counted as a feature of an item depends, in part, on a person's previous experience, as well as on various perceptual constraints.

Importantly, however, concept learning consists not only of identifying the actual given features and using them to categorize new instances, but also of understanding how the concept coheres. Observable features are often related through more abstract, nonobservable features, and the concept may cohere at this more abstract level (see, e.g., Medin \& Ortony, 1989; Michalski, 1983; Mooney, 1993; Wisniewski \& Medin, 1991, 1994). Thus, the link from the observable features to the more abstract features is critical to understanding how a concept coheres. For example, a particular behavior may be linked to different abstract features (e.g., riding a bike to work might be linked to the abstract features "athletic" or "environmentally concerned"). Such different abstract features, if invoked, would likely have strong effects on how one dealt with and thought about the individual, and perhaps, more generally, how one deals with the members of that group (e.g., whether one thinks members of the group might donate money to an environmental cause). Thus, the link from the observable feature to the abstract feature is critical to understanding how the category coheres and to understanding what is important about the category both in the sense of what features are likely to indicate category membership and what features are likely to be reasonable inferences about a known member of the category.

Wisniewski and Medin $(1991,1994)$ have demonstrated the importance of abstract features in category learning and in interpreting features. Their participants viewed drawings created by children, and were given differing information about the children who created the drawings. For example, one group of participants was told that some of the drawings were done by creative children and that the others were done by children who were less creative. The other group of participants was told that some of the drawings were done by children from the city and the others were done by children from farms. In each case, the participants were to learn which drawings were done by which group of children. Wisniewski and Medin suggested that when people learn categories they induce features at multiple levels of abstraction. Thus, the participants were expected to (and did) form expectations about the drawings on the basis of what they had been told about the children who had created them. For example, the participants believed that the drawings done by creative children should show more detail than the drawings done by less creative children. Similarly, the participants expected that drawings done by farm children and city children would differ on the type of clothing drawn.

On the basis of their expectations for abstract features, the participants showed two effects that are important for the present paper. First, the abstract features activated by the participants' expectations about the drawings (e.g., being detailed) led them to consider some very different physical features as being basically the same; that is, certain features were all considered as evidence of detail in the drawings. For example, buttons, shirt cuffs, shoelaces, eyebrows, and eyeglasses might all have been considered as examples of "detail" in a set of drawings. Second, the participants sometimes interpreted physically identical features in the drawings as two completely different features. For example, one participant, expecting detail in the drawings, interpreted one drawing as having buttons down the front of the person's shirt, thus indicating detail-evidence that a creative child was the artist. Another participant, who was looking for city/farm differences, on the other hand, interpreted exactly the same physical stimulus (a series of circles down the front of the shirt) in the same drawing as a tie, thus indicating city clothes-evidence suggesting a city child was the artist. The participants' knowledge about the possible source of the drawings helped them interpret the drawings and identify the features of the drawings that should be used as evidence for which categories they belonged to. Thus, the expectations for abstract features, arising from the participants' knowledge of the kind of children who drew the pictures, affected what features were identified in the physical stimulus.

However, there are likely to be many cases in which one does not have the requisite knowledge to interpret the features as evidence for a more abstract feature. Thus, many cases of category learning and feature interpretation remain to be explained, above and beyond interpretations based on knowledge or theories about the categories. In the present experiments, we investigated another possible mechanism for how abstract features might be activated and how they might affect the feature interpretation. This mechanism involves the availability of particular instances within a category. In particular, one might be able to interpret features by comparing them with available members of the category. Similarities between the compared instances might give rise to an interpretation of a particular feature as evidence for an abstract feature that captures the similarity between the instances. This is a mechanism that might be very useful even if one has a large amount of background knowledge (e.g., if background knowledge did not lead to a particular expectation about abstract features), and the mechanism would certainly be helpful if one did not have much background knowledge. Importantly, this mechanism provides a way of bootstrapping one's way to a feature interpretation when one does not have a lot of prior experience in a categorical domain, and thus when one does not have the requisite background knowledge to interpret the features. The methods of the present experiments allow us to rule out the effects of background knowledge, per se, while showing the effects of differential availability on feature interpretation.

In the present experiments, the participants were not given category labels that would allow them to make use of their prior knowledge. The participants had background knowledge (presumably) that would enable them to link the given and the abstract features; that is, they 
would be able to link the feature of riding a bike to the features athletic and environmentally concerned. However, in all of the present experiments (as in, probably, many cases of learning new categories), the participants' background knowledge was consistent with either interpretation; thus background knowledge per se could not have helped the participant to settle on a particular interpretation of the target features. For example, consider the feature rides a bike to work. Our general knowledge makes a connection between this feature and a number of different possible abstract features, such as environmental concern, interest in exercise, or perhaps, an interest in saving money. In the present experiments no extra background knowledge was available to the participants that would allow them to choose any one of these possible interpretations. What led the participants to a particular interpretation in the present experiments was the availability of other exemplars that could give rise to different abstract features. For example, if a previous athletic behavior is highly available, will this lead to an athletic interpretation of rides a bike to work? On the other hand, if a previous environmentally concerned behavior is highly available, will this lead to an environmental interpretation of rides a bike to work? As noted above, this question is important because there are likely to be many cases in which one simply does not have enough background knowledge to interpret the features. Thus, feature interpretation via comparison with other instances allows one to interpret the observable features with respect to more abstract features, which is likely to help the learner understand how the category coheres.

In the present experiments, we investigated whether the choice among such alternative interpretations can be driven by the availability of other category exemplars that have observable features that suggest a particular abstract feature. In addition, we investigated whether such interpretation effects would affect the learners' overall evaluations of the categories. To the extent that activating common abstract features provides some coherence to the category, one would expect activation to influence the interpretation of features and the representation of the category. To examine these issues, the experiments included three different ways of manipulating the availability of category members: spatial grouping, presentation order, and remindings to similar instances. In Experiment 1 , we presented category members in two different spatial groupings and showed that what people thought was important about the group depended on the spatial groupings of the category members. Encountering features in close proximity to other features that supported a particular interpretation led learners to interpret the features as being consistent with each other. Experiment 2 was analogous to Experiment 1, except that we manipulated the order of presentation (temporal grouping) instead of spatial grouping. In Experiment 2, it was shown that recent exemplars containing features sup- porting a possible interpretation were likely to affect how people interpreted a new feature.

In Experiments 3 and 4, we investigated whether feature interpretation would occur when a person was reminded of an earlier exemplar, even if the reason the person was reminded of the earlier example had nothing to do with the interpretation itself. Previous research (Ross, Perkins, \& Tenpenny, 1990; Spalding \& Ross, 1994) had shown that such remindings during category learning influenced what features were believed to be important for a category. In Experiments 3 and 4, we went beyond the previous work by showing that such remindings affected not only whether people thought a given feature was important, but how they interpreted that feature. To return to our previous example, suppose a person who rides a bike to work reminds you of someone athletic, perhaps because they are both extremely blond. Does rides a bike to work get interpreted as indicating athleticism? What if a person who rides a bike to work reminds you of someone who is very concerned about the environment, perhaps because both had very red hair? Would rides a bike to work get interpreted as indicating environmental concern? Obviously, hair color has little to do with athleticism or environmental concern. Nevertheless, once you are reminded of the person, the interpretation of rides a bike to work may be driven by the features of the highly available person.

In sum, in the present experiments, we attempted to determine whether observable features would be interpreted as indicating different abstract features, depending on which abstract features were suggested by the observable features of other highly available instances.

\section{EXPERIMENT 1}

In this experiment, we examined whether observed features might be interpreted differently at a more $a b$ stract level as a function of their grouping with other features. Does the activation of an abstract feature by some presented items in a group influence the interpretation of another item in that group? Consider the feature elected to the county recycling board. Although the event is clear, what it tells us about the person is not. Suppose you learned that this person was in the same club as two other people, one who ran for mayor to start a city homeless shelter and another who was the chairperson of the state committee on university funding. Taken together, there is a politically active theme to these features. However, if elected to the county recycling board is presented with rides a bike to work and paid for expensive new low-energy lights in his businesses, then the county-recycling-board feature seems less political and more environmental. Similarly, if ran for mayor to start a city homeless shelter occurred with a number of other features relating to charity activities, then it might seem less political and more charity oriented, and if chairperson of the state committee on 
university funding occurred with other features that had education-oriented interpretations, then it would seem less political and more concerned with education.

All of the target features in this experiment could be interpreted as being related to two different abstract features (such as politically active or environmentally concerned). The availability of other features during encoding of the target features was manipulated by spatially grouping different category members. All of the participants saw exactly the same target features, but the features were spatially grouped to promote one set of abstract features (environmental, charitable, higher education) or another (political, wealthy, athletic). The question of interest was whether such a manipulation of availability would lead to different interpretations of the target features, which would presumably be due to the activation of different abstract features.

\section{Method}

Participants. Forty University of Iowa undergraduates participated for course credit.

Materials. In this experiment, nine critical observable features were organized around a set of six abstract features. The materials are presented in Table 1. As can be seen in Table 1, there were descriptions of nine people, and two features were listed for each person described. In each presentation condition, the nine descriptions were divided into three groups of three descriptions each. Which descriptions were grouped together was the only experimental ma- nipulation. For each description, the first feature was the critical one. A separate sheet of paper listed a set of characteristics that the participants rated for typicality for each group.

Procedure. The participants were instructed that they would be reading some brief descriptions of people who all belonged to a particular club. They were asked to read the descriptions and form an impression of each person and of the club as a whole. They were informed that they would be asked some questions after reading the descriptions. The participants saw all of the descriptions at one time, on one sheet of paper. The participants spent approximately 1 min reading the descriptions. Next, the participants were given a sheet of paper that listed 10 characteristics, including "Concerned about the environment," "Charitable," "Involved in higher education," "Politically active," "Wealthy," "Athletic," and four unrelated filler characteristics. The participants were asked to rate to what extent each of the items was characteristic of the group on a 7-point scale, where 1 was not at all characteristic and 7 was extremely characteristic. The participants' ratings of these items were compared to see whether the grouping of the descriptions affected how the participants thought about the group.

Design. The spatial grouping of the instances during the initial study period was the only manipulation. Half of the participants received Grouping 1 and half received Grouping 2. Grouping 1 was predicted to lead the participants to interpret the group as being environmentally concerned (county recycling board, paid for lowenergy lights, rides a bike to work), charitable (ran for mayor to start a homeless shelter, donated $\$ 100,000,10 \mathrm{k}$ fund-raisers), and involved in higher education (state committee on university funding, founded an endowed professorship at Harvard, the college football team), whereas Grouping 2 was expected to lead participants to interpret the group as politically active (county recycling board, ran

Table 1

Materials From Experiment 1

Environmental
Bill was elected to the county recycling board and subscribes to a newsmagazine.
Tom paid for expensive new low-energy lights in his businesses and visited a museum.
John rides a bike to work and has coffee with friends.
Charitable
Mark ran for mayor to start a city homeless shelter and enjoys plays.
Sam donated $\$ 100,000$ for a youth shelter and eats Indian food.
Kevin runs in $10 \mathrm{k}$ fund-raisers and took his daughter to the library.
Higher Education
Greg is the chairperson of the state committee on university funding and reads mysteries.
Jim founded an endowed professorship at Harvard and plays guitar.
Larry plays for the college football team and likes movies.
Political
Bill was elected to the county recycling board and subscribes to a newsmagazine.
Mark ran for mayor to start a city homeless shelter and enjoys plays.
Greg is the chairperson of the state committee on university funding and reads mysteries.
Wealthy
Tom paid for expensive new low-energy lights in his businesses and visited a museum.
Sam donated $\$ 100,000$ for a youth shelter and eats Indian food.
Jim founded an endowed professorship at Harvard and plays guitar.
Athletic
John rides a bike to work and has coffee with friends.
Kevin runs in $10 \mathrm{k}$ fund-raisers and took his daughter to the library.
Larry plays for the college football team and likes movies.
Note--The labels "Environmental," "Charitable," and so forth were not presented to the par-
ticipants.


for mayor to start a homeless shelter, state committee on university funding), wealthy (paid for low-energy lights, donated $\$ 100,000$, founded an endowed professorship at Harvard), and athletic (rides a bike to work, 10k fund-raisers, the college football team). Each participant saw only one grouping of instances.

\section{Results and Discussion}

For each participant, the ratings for the environment, charity, and education test items were summed, as were the ratings for the political, wealthy, and athletic test items, and the difference between the predicted and nonpredicted interpretations was computed. Overall, the participants rated the environment, charity, and education test items as being more characteristic of the group than the political, wealthy, and athletic test items. This general bias for some of the test items was unrelated to the hypothesis, which was about the effect of relative preferences among the test items based on the order manipulation. Although the counterbalancing across the participants means that this bias did not affect the mean difference score, it did add to the variability in the difference scores. To remove this variability, we corrected each of the participant's difference score by subtracting the mean difference score of the condition and adding the grand mean difference score. This correction did not affect the mean difference, but did reduce the variability, essentially taking out the variability associated with the overall bias toward the environment, charity, and education test items. On average, the grouping manipulation increased the rating of the predicted interpretations by $1.03\left[M_{1}=14.13, M_{2}=\right.$ $13.10 ; t(39)=2.03, S E M=.507, p<.05]$. Results for 27 participants showed the predicted advantage, and those for 13 did not $(p=.02)$.

The grouping of the descriptions affected what participants thought of the group. The most likely explanation for this was that the spatial groupings affected what abstract features were activated and used to interpret the critical features. The observable features were interpreted in a way congruent with the shared interpretation of other observable features within the spatial group, even though all of the observable features could have provided evidence for two abstract features (e.g., runs in $10 \mathrm{kfund}$ raisers could have been evidence for either charitableness or athleticism).

\section{EXPERIMENT 2}

The finding from Experiment 1 was extended in Experiment 2 in two important ways. First, the presentation order of the category members was used to manipulate the availability of features, thus adding some generality to the findings from Experiment 1 . Other results have shown that presentation order can have large effects on the category representation by differentially weighting observable features (e.g., Elio \& Anderson, 1981, 1984; Medin \& Bettger, 1994). Extending the feature-weighting idea to the idea of activating abstract features, we predicted that the participants would be likely to interpret a target feature in a way congruent with other recent features.
Second, in Experiment 2, we tested specific features to determine whether the participants did in fact interpret the feature in the way that we have claimed, rather than test the overall impression of the group (as in Experiment 1). Although it seems likely that in Experiment 1 the features were interpreted, and thus changed the impression of the group as a whole, in Experiment 2 we tested the interpretation of the features directly.

\section{Method}

Participants. Thirty-six University of Iowa undergraduates participated for course credit.

Materials. The participants were again shown descriptions of fictitious people. The descriptions are presented in Table 2. Two kinds of tests, using open-ended questions and rating scales, were included. The open-ended-question tests had descriptions of Larry or Mark, along with two questions on sheets of paper. The first question asked for an overall impression of the person described at the top of the page, and the second question asked the participant to provide a new feature that might be true of the person described at the top of the page. The rating-scale tests were also presented on sheets of paper with the descriptions of Larry or Mark. Each page had a rating scale for one of the interpretations of the critical feature in the description. For example, one sheet had the Larry description at the top and a scale from 1 to 7 for indicating how athletic he was, and the other sheet had the same description of Larry and a rating scale for indicating how charitable he was.

Design. The only experimental manipulation was whether participants received Order 1 or Order 2. As can be seen in Table 2, the only difference between Order 1 and Order 2 was that the descriptions of Mark and Larry switched positions. The rationale for this manipulation was that the critical feature was expected to be interpreted as congruent with other recent, related features. For example, the feature installed a solar water heater in the description of Mark was expected to be interpreted as evidence for environmental concern when it occurred just after features such as started a compost heap in Bill's description and had insulation put in his attic in Tom's

Table 2

Materials Used in Experiment 2

Order 1

John subscribed to Newsweek and had coffee with friends.

Bill started a compost heap and collected clothes for the homeless.

Tom had insulation put in his attic and organized a food drive.

**Mark installed a solar water heater and visited an art museum.

James got new eyeglasses and flew to Baltimore on a business trip. Sam built a storage shed for tools and played basketball.

Greg fixed his doorbell and went swimming.

** Larry went to the library and ran in a $10 \mathrm{k}$ fund-raiser.

Gary went to a play and had dinner at an Indian restaurant.

Order 2

John subscribed to Newsweek and had coffee with friends.

Bill started a compost heap and collected clothes for the homeless.

Tom had insulation put in his attic and organized a food drive.

**Larry went to the library and ran in a $10 \mathrm{k}$ fund-raiser.

James got new eyeglasses and flew to Baltimore on a business trip.

Sam built a storage shed for tools and played basketball.

Greg fixed his doorbell and went swimming.

**Mark installed a solar water heater and visited an art museum. Gary went to a play and had dinner at an Indian restaurant.

Note-The descriptions of Larry and Mark were not asterisked in the materials presented to the participants. 
description. However, when it occurred just after the feature built a storage shed for tools in Sam's description and fixed his doorbell in Greg's description, the feature installed a solar water heater was expected to be interpreted as indicating that Mark was handy. Similarly, the critical feature ran in a lok fund-raiser in the description of Larry was expected to be interpreted as evidence for charity when it followed the collected clothes for the homeless feature in Bill's description and the organized a food drive feature in Tom's description. However, ran in a $10 \mathrm{k}$ fund-raiser was expected to be interpreted as athletic when it followed the played basketball feature in Sam's description and the went swimming feature in Greg's description.

Procedure. The participants read through the descriptions, one at a time, with $15 \mathrm{sec}$ allotted for each description. They were told that each of these people belonged to the same club, and they were asked to form an impression of each person and of the club overall. The participants were told that they would be asked some questions about the people and the club later. After reading the descriptions, each participant received a test sheet with a description of either Mark or Larry along with the two questions described above. On each of the next two pages, the participants saw the same description with a rating scale underneath. Each participant was tested on both Larry and Mark. Half of the participants got the Larry tests first, and the other half got the Mark tests first. Within each group, half of the participants got one order of the rating scales (e.g., charitable, then athletic) and the other half of the participants got the reverse order of the rating scales (e.g., athletic, then charitable).

\section{Results}

Two independent judges scored each question for all participants (the judges were blind to the participants' conditions). Each answer was scored as strongly indicating one of the interpretations, as weakly indicating one of the interpretations, or as indicating each interpretation equally (including not indicating either interpretation). Then, if a strong interpretation matched the prediction, a score of +1 was assigned; if it mismatched the prediction, a score of -1 was assigned. Weak interpretations were given $+1 / 2$ or $-1 / 2$ if they matched or mismatched the predicted interpretation, respectively. Answers that favored neither interpretation were assigned a score of 0 . In case of disagreement, the answer was assigned the value closer to 0 . Overall, about $64 \%$ of the participants' answers were coded as strong interpretations, and about $4 \%$ were coded as weak interpretations. The remaining items were scored as 0 . Our coding was quite conservative. Strong interpretations were those that clearly matched only one of the expected interpretations (e.g., running in a $10 \mathrm{k}$ fundraiser for athletic reasons, not charity reasons), such as, "works out at healthclubs" or "Larry is a fitness freak." Weak interpretations were those that seemed more related to one interpretation but not quite as obviously as the strong interpretations, such as "Very fit. I'm not sure if he is running particularly for the fundraiser or just for the 10k." Answers that favored neither interpretation, either by being unrelated (e.g., "interested in art") or by including both interpretations equally, received a score of 0 . Agreement between the scorers was $95 \%$.

The data were analyzed using both a simple nonparametric sign test (with each participant counted as plus, minus, or zero) and a $t$ test of the actual scores. For Question 1 , the general description, 18 participants gave the predicted interpretation and 7 gave the opposite interpretation $(p<.05)$. The mean score was +.36 (of a possible 2) $[t(35)=2.12, S E M=.17, p<.05]$. For Question 2, which asked the participants to generate new features, 22 participants gave features consistent with the predicted interpretation and 7 gave features consistent with the opposite interpretation $(p<.01)$. The mean score was +.53 (of a possible 2) $[t(35)=2.59, S E M=.205, p<.05]$.

For each participant, the rating given to the predicted interpretation was compared with the rating given to the nonpredicted interpretation for both the Larry and Mark tests. The predicted-nonpredicted differences were summed for each participant. These summed differences were then analyzed. For example, consider a participant who received Order 1. For such a participant, Mark was expected to be judged as being concerned about the environment rather than handy, and Larry was expected to be judged as being athletic rather than charitable. So, for this participant, the rating of Mark's handiness was subtracted from the rating of his concern for the environment, and the rating of Larry's charitableness was subtracted from the rating of his athleticism. The differences for the Mark ratings and the Larry ratings were then summed. For the summed differences, 22 participants were in the predicted direction and 7 were in the opposite direction $(p<.01)$. The mean predicted-nonpredicted difference was $.92\left[M_{1}=4.61, M_{2}=5.53 ; t(35)=3.82\right.$, $S E M=.24, p<.01]$.

\section{Discussion}

Manipulating the order in which the instances were presented led the participants to interpret the same observable feature as evidence for differing abstract features. These abstract features were not "minor variations"-for example, one's expectations for a member of a group that is thought of as athletic are likely to be very different from one's expectations for a member of a group that is thought of as environmental. The fact that the very same observable features support such different abstract features shows how important the effects of feature interpretation are likely to be in determining how one deals with a category (e.g., the kinds of inferences one is likely to make). Taking Experiments 1 and 2 together, we can conclude that observable features may be interpreted as evidence for different abstract features, depending on what other observable features are highly available. This conclusion holds whether the availability is due to spatial grouping or to temporal recency and whether the test is for the individual feature (Experiment 2) or for the category as a whole (Experiment 1).

Taken in a broader perspective, these results are consistent with a variety of earlier findings. For example, although categorization models have tended to ignore these kinds of issues, it has been clear, at least since Asch (1946), that the order in which information is presented can af- 
fect the interpretation of that information. Thus, the first two experiments perhaps serve primarily to remind categorization theorists that they cannot escape these effects and cannot assign these effects to some "lower level" systems. Instead, categorization theories must include, in one way or another, mechanisms that give rise to the (interpreted) feature description that is then used to represent the category member. It seems clear that interpreting the features is indeed an important part of category learning.

In the first two experiments, we varied the availability of items in very broad ways, by manipulating spatial grouping and recency. In the next two experiments, we examined whether feature interpretation could be driven by comparisons between an item and a highly similar previous item. These experiments had two important purposes. First, we intended to show more directly that the availability of particular instances would affect the interpretation of an observable feature, rather than more general information about the category as a whole. For example, one might believe that having seen (recently) two category members that have athletic features makes a person more likely to interpret ran in a $10 \mathrm{k}$ fund-raiser as athletic because when the target feature is encountered, these earlier features have already made the learner believe that the category might be of athletic people rather than because of the availability of those earlier items per se. That is, the effect of the order manipulation on feature interpretation might be mediated by the effect of the order manipulation on the category itself, even before the critical item is encountered. In Experiments 3 and 4, however, we manipulated the similarity between the target instance and previous instances, so that a very similar previous instance would likely be highly available when the learner encountered the target item. In these experiments, we could ensure that the availability of the particular earlier instance would be driving the interpretation, rather than the effect's being mediated by beliefs about the category as a whole. Because features consistent with both interpretations have been seen when the learner encounters the critical item, there is no reason for the participants to favor one interpretation or the other before seeing the critical item. To accomplish this manipulation, we used a very small set of items and a very minimal change between conditions (only one feature differed between conditions) in Experiments 3 and 4. The simple nature of the materials allowed us to be sure that the availability of the earlier item, owing to the similarity to the target instance, would drive the interpretation. Thus, by manipulating the availability of the particular instance directly, we intended to demonstrate more conclusively that availability could drive feature interpretation and to rule out a mediating effect of the category itself before the critical example was encountered. Put another way, in the earlier experiments, the abstract features (such as athletic) might well have been activated before the critical instance was presented, whereas in Experiments 3 and 4 any differential activation of the abstract features would have to be due to the comparison of the critical instance with an earlier item.

Second, we intended to tie the present work more closely to earlier research on category learning, in which subjects have often made use of experience with highly similar prior instances during category learning. Ross et al. (1990) showed that when one is reminded of a prior instance during category learning, similar features of the compared instances become more important or central to the category than do other equally frequent features. Spalding and Ross (1994) showed that such instance comparisons during learning affect later categorization, typicality ratings, subjective frequency, and ease of learning about correlated features. In short, comparisons between a new instance and a highly similar old instance had large effects on what was learned about the concept (see also Medin \& Bettger, 1994; Medin \& Florian, 1992). In general, features that were common to the compared instances were given more importance than features that were not common to the compared instances. However, this earlier work examined the weighting of observed features and not the effect on the interpretation of the observed features with respect to more abstract features.

Ross and Bradshaw (1994) showed that interpretation of ambiguous texts could be driven by a salient superficial similarity to other text passages. However, there were many differences between the present experiments and those of Ross and Bradshaw. Most importantly, the passages in Ross and Bradshaw were truly ambiguous in the sense that the participants could not understand the passages unless they interpreted the ambiguous portions in some way. In Experiments 3 and 4 (as in Experiments 1 and 2), there was no need for the participants to do any interpretation in order to do the experimental task. Nevertheless, as we have argued in the introduction to this paper, understanding how a category coheres is a critical component of learning about real concepts, and learning to link observed features to more abstract features is important when one is learning how the concept coheres. It therefore seemed highly likely that the participants would interpret the observable features in terms of an abstract feature, in order to provide more coherence to the categories they were learning. Thus, it seemed highly likely that comparison with previous category members could drive interpretation of observable features (see also Medin, Goldstone, \& Gentner, 1993, Experiment 1).

\section{EXPERIMENT 3}

In Experiment 3, we investigated whether observable features would be interpreted as consistent with abstract features suggested by comparison with similar instances. This experiment followed the method of Ross et al. (1990). The participants learned about a set of instances belonging to a pair of categories (Group X and Group Y). They then encountered a test instance that was intended to remind them of one of the studied instances. The test instance had one critical feature that could be interpreted 
Table 3

Example Materials From Experiments 3 and 4

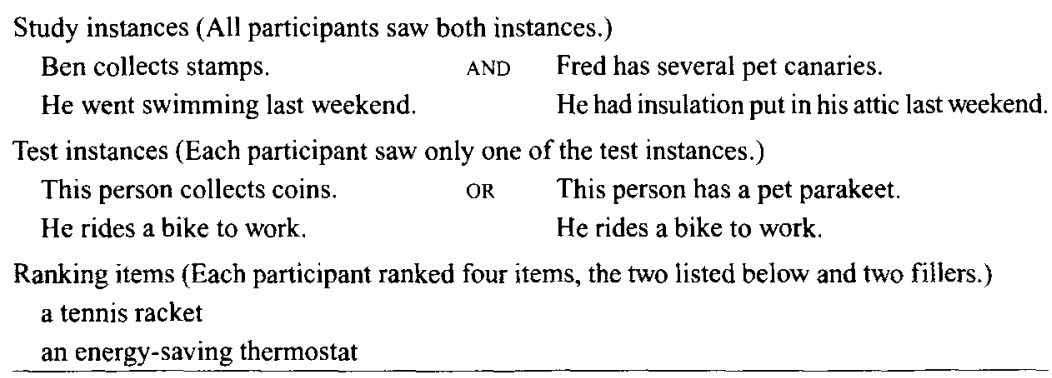

Note-These are example materials from only one group learned by the participants.

in two different ways, each of which was consistent with a feature present in one of the study instances. The participants were expected to interpret the critical feature of the test item as being congruent with the instance of which they were reminded.

An example of the stimuli may make the rationale clearer (see Table 3). The participants learned about two members of Group X (and two members of Group Y, though they are not included in this example): "Ben collects stamps. He went swimming last weekend." "Fred has several pet canaries. He had insulation put in his attic last weekend." After learning the study examples, they were given one of two test instances that had to be categorized as either Group X or Group Y. For the test instance that was intended to be categorized as a member of Group X, the participants saw either This person collects coins, He rides a bike to work, or This person has a pet parakeet, He rides a bike to work. The first test instance above was intended to remind the participants of Ben, and the second was intended to remind the participants of Fred. If reminded of Ben, the participants were expected to interpret rides a bike to work as athletic (because of Ben's swimming), and if reminded of Fred, they were expected to interpret rides a bike to work as environmental (because of Fred's insulation). Note that in each case, the test instance in the preceding example should be categorized as a member of Group X. The only manipulation was which previously learned Group X member (Ben or Fred) the participant was reminded of. Thus, the point here was that the critical feature was interpreted differently depending on which earlier Group X member the participant was reminded of, even though the test instance was categorized as a member of Group $\mathrm{X}$ in both cases. The effect of the interpretation was examined in a second test, in which the participants ranked a set of items on the basis of how likely the items were to be purchased by a member of Group X. There were two items critical to this test, one related to an athletic activity (tennis racket) and one related to environmental concern (an energy-saving thermostat). The participants who were reminded of Ben (who went swimming) were expected to choose the tennis racket as a more likely purchase, and participants reminded of Fred (who had insulation put in his attic) were expected to choose the energy-saving thermostat as the more likely purchase.

Thus, this experiment showed whether a comparison of the critical item to a previous example could activate an abstract feature that, in turn, would drive feature interpretation of the critical item. In this experiment, more general effects (i.e., effects mediated by the category itself) were unlikely to drive the interpretation, because the earlier items were controlled with respect to any differential interpretation (i.e., more general effects would not lead to any difference between the conditions). Thus, this experiment provided the most stringent test of whether the availability of a particular instance could drive feature interpretation. In order to rule out other possible explanations, we attempted to pare this experiment down to the bare essentials. Therefore, the materials in this experiment were very simple: each category had only two members at study and only one more at the first test, and the whole experimental manipulation consisted of changing a single feature on each of the first test instances.

\section{Method}

Participants. Forty undergraduates from the University of Illinois participated for course credit.

Materials. Each participant was given a booklet in which the study instances had been printed. There were eight different study booklets, corresponding to eight different possible orders of the study items, under the constraint that Group X and Group Y items had to alternate. An equal number of participants was assigned to each booklet. Each study instance was presented on a separate page of the booklet. A second booklet contained the test instances (one for Group X and one for Group $Y$ ) and lists of items to be ranked for each group, all on separate pages. An example of the materials used is given in Table 3.

Procedure. The participants were tested in small groups. The procedure consisted of a study phase, a first test of categorization of new instances, and a second test in which the participants ranked certain items in terms of how likely a member of a given category was to buy them. In the study phase, the participants were told that they would be seeing descriptions of four people and that they would be asked some questions about them later. The participants saw each description for $20 \mathrm{sec}$ and were asked to try to form an 
image of the person being described. The participants then saw each description again for an additional $20 \mathrm{sec}$ and were again asked to create an image of the person being described. These instructions were intended to ensure that the participants would be able to remember the study items reasonably well. The participants were then told that two of the descriptions belonged to Group $X$ and two to Group Y. They were shown the descriptions for $20 \mathrm{sec}$ again, this time with the group name written below the description.

After completing the study phase, the participants were given a test booklet and told that they would be seeing some new descriptions that they should categorize as belonging to Group X or Group Y. They saw two test instances (one from Group $X$ and one from Group $Y$ ) and wrote down the group to which the person belonged. The participants were then shown the test instances again for $15 \mathrm{sec}$ with the correct group label written below the description. The participants then gave their general impressions of the groups. After giving their general impressions, they were given two lists of items (one for Group X and one for Group Y) and were asked to rank how likely each item was to be purchased by a member of the given group.

Design. The only manipulation in this experiment involved one feature of the test instances. The test instances were designed to remind the participants of one of the study instances, shown in Table 3. Within each category, half of the participants were intended to be reminded of each study instance (i.e., half of the participants were intended to be reminded of Ben and half of Fred on the Group X test instance) by including a salient feature that was highly similar to one of the study instances (e.g., collects coins to remind them of Ben, who collected stamps). All participants saw the same critical features in the test instances (see Table 3). Thus, any differences in the participants' rankings would have to be due to the salient feature of the test instances. Note that these salient features were unrelated to the items that the participants later ranked (e.g., collecting coins is not related to either a tennis racket or an energy-saving thermostat).

\section{Results and Discussion}

Twelve participants were excluded from the analysis either for failure to follow instructions on the ranking task or for incorrectly categorizing a first-test item. Incorrectly categorizing a first-test item suggested that the person was not reminded of the appropriate study item, making the ranking data meaningless with respect to our hypothesis. The remaining participants' rankings were examined for differences between the feature related to the expected interpretation and the feature related to the nonexpected interpretation. For example, a participant who was reminded of Ben (who went swimming) was expected to rank the tennis racket as a more likely purchase than the energy-saving thermostat, while the preference should have reversed for a participant reminded of Fred (who had insulation put in his attic). The participants ranked four items for each category. Thus, if the expected item was ranked first and the nonexpected item was ranked fourth, which would mean ranking it as worse than the unrelated fillers, there would be a difference of three ranks in each category, yielding a maximum possible difference of six ranks across the two categories (if both critical items were ranked above the fillers, the maximum rank difference would be two). The expected interpretation items $(M=3.14)$ had an average advantage of 1.32 ranks over the nonexpected interpretation items $[M=4.46, t(27)=$ 2.87, SEM $=.46, p<.01]$. Seventeen participants gave higher ranks to the expected items and 4 gave higher ranks to the nonexpected items $(p<.01)$. The general impressions given by the participants often simply repeated features (particularly the salient features) of the group members and were thus not very helpful in determining how the participants interpreted the critical features.

What aspects the participants thought were important about these groups depended on which study instances they were reminded of when they encountered the test instances. One interpretation of the results is that the comparison of instances led the participant to interpret the critical observable feature as being consistent with the abstract feature. This comparison manipulation led the participants to choose very different later features as being consistent with the category. In the next experiment, we used a very different measure of the comparison effect by directly asking the participants about their feature interpretations.

\section{EXPERIMENT 4}

The goal of Experiment 4 was to investigate more directly whether being reminded of a previous instance affected the interpretation of the critical feature. The method was the same as in Experiment 3 through the presentation of the test item. After the participants categorized the test items and saw the correct group listed with each item, they were asked a series of three questions aimed at assessing whether they had interpreted the critical features as predicted. The first question asked them for a general impression of the person described in the test item. The second question asked the participants to list another feature that might be true of the person (a new feature that did not occur during the experiment). These two questions were the same as those in Experiment 2. The third question asked directly for an interpretation of the critical feature (e.g., Why did this person ride a bike to work?). Although the third question did explicitly ask the participants to provide an explanation for the critical feature, the first two questions did not. That is, if a person had not interpreted the feature as signifying athleticism, then it would have been perfectly reasonable to give impressions and feature listings that did not include any athleticism, by focusing on the reminding feature (e.g. "this person likes to collect things"), interpreting the feature in some other way (e.g., "this person likes to do outside activities such as bike riding"), or just giving an overall evaluative rating (e.g., "this person seems boring"). Thus, even though any question had the potential for forcing people to make inferences that they had not made before the question was asked, the first two questions were chosen to allow answers that did not require activating the particular abstract features in common between the study and test instances.

In addition, in Experiment 3, although the number of participants in the analysis who received the various orders of study items was roughly equal, some of the or- 
ders were slightly more frequent than others, owing to the participants' making errors on the first test items. In Experiment 4, the participants who made errors on the first test items were replaced in the design to ensure that the results were not affected by differences among the orders of the study items.

\section{Method}

Participants. Forty undergraduates from the University of Illinois participated for course credit. Fourteen additional participants incorrectly categorized a first test item and were replaced in the design.

Materials. The study and test materials were the same as in Experiment 3 , except that the participants were also given a page that included the test instance, correct category label (e.g., Group X), and three questions to be answered. The ranking tests from Experiment 3 were not used.

Procedure. The study and test procedures were identical to those in Experiment 3, except that the participants were asked to answer three questions about the test instances after they had categorized the test instances. Question 1 asked the participants to give a general impression of the person described in the test instance. Question 2 asked the participants to give a new feature (a feature not used previously in the experiment) that they thought was likely to be true of the person described in the test instance. Question 3 asked directly for an interpretation of the critical feature of the test instance (e.g., "Why does this person ride a bike to work?").

\section{Results and Discussion}

Both authors scored each question for all participants (the authors were blind to the participants' condition). As in Experiment 2, each answer was scored as strongly indicating one of the interpretations, as weakly indicating one of the interpretations, or as indicating each interpretation equally (including not indicating either interpretation). Then, if a strong interpretation matched the prediction, a score of +1 was assigned; if it mismatched the prediction, a score of -1 was assigned. Weak interpretations were given $+1 / 2$ or $-1 / 2$ if they matched or mismatched the predicted interpretation, respectively. Other answers were assigned a score of 0 . The authors agreed on $94 \%$ of the answers given by the participants. In case of disagreement, the answer was assigned the value closer to 0 . Overall, about $63 \%$ of the participants' answers were coded as strong interpretations, and about $12 \%$ were coded as weak interpretations. The remaining items were scores of 0 . Again, our coding was quite conservative. Strong interpretations were those that clearly matched only one of the expected interpretations (e.g., riding the bike for exercise reasons vs. for environmental reasons), such as "gets her exercise by riding a bike" or "this person jogged 3 miles this morning. He is very active and into exercise." Weak interpretations were those that seemed more related to one interpretation but not quite as obviously as the strong interpretations, such as "health conscious" or "this is a healthy person" (weak because we cannot be completely sure they are referring to the exercise of bike riding). Scores of 0 were assigned to all answers that favored neither interpretation, either by being unrelated, such as "eccentric" or "doesn't socialize much," or by including both interpretations equally.
Overall, the data provided strong evidence that the participants interpreted the features as predicted. For Question 1, which asked for a general description of the test instance, 20 participants gave the predicted interpretation and 9 gave the opposite interpretation ( $p=$ .06). The mean score was +.43 (of a possible $+2 ;+1$ from each category) $[t(39)=2.53, S E M=.17, p<.02]$. For Question 2, which asked the participants to generate new features, 21 participants gave features consistent with the predicted interpretation and 7 gave features consistent with the opposite interpretation $(p<.02)$. The mean score was +.53 (of a possible 2) $[t(39)=3.31, S E M=$ $.16, p<.01]$. For Question 3, which specifically asked the participants to interpret the critical features, 22 participants gave the predicted interpretation and 7 gave the opposite prediction $(p<.01)$. The mean score was +.44 (of a possible 2) $[t(39)=2.75, S E M=.16, p<.02]$.

The responses of the participants were consistent with the idea that they were interpreting the critical features of the test instances in a way that was congruent with the features of the instance of which they were reminded. Because the dependent measure involved probing directly for information about the test instance, these results cannot rule out the possibility that the interpretation was derived at test from other information encoded as a function of the reminding manipulation (e.g., a heightened availability of the reminded study instance, as tested and rejected in Ross et al., 1990). However, in either case, the interpretation provided at test did change as a function of the reminding manipulation. Taken together, the results of Experiments 3 and 4 indicate that instance comparisons can strongly affect how observed features are interpreted. In addition, given that the ranking test of Experiment 3 asked generally about members of the group, the interpretation of the critical features can affect what is believed to be important about the category as a whole.

\section{GENERAL DISCUSSION}

Across the four experiments presented here, we see strong evidence that observable features are interpreted with respect to abstract features activated by highly available category members. The interpretation effect holds up over different material sets, over different study tasks (such as whether the learner must learn one category or two), over different manipulations to make the instances available (spatial grouping, presentation order, and similarity), and over different dependent measures. It is clear that people interpret individual observable features as evidence of more abstract features (e.g., interpreting rides a bike to work as evidence of environmental concern) based on other highly available instances of the category, and that the interpretation affects the category as a whole. In the rest of this discussion, we will focus on the implications of these results for future work on feature interpretation and concept research more generally. 


\section{Implications for Future Research}

The most general implication of our results is that feature interpretation may be much more common than previously thought. Feature interpretation is important at many different levels of concept learning. Four aspects of these results need particularly to be highlighted: the idea of levels of features, the relationship between feature interpretation and the broader notion of inference, the generality of the present results, and the possibility of contrast effects in interpretation as well as the assimilation effects that we have presented.

Levels of features. It seems clear that learners will induce features at higher levels of abstraction in order to make the category cohere better, and that the induced abstract features can affect what the learners think about individual category members and the category as a whole. The observable features in the present experiments could be taken as evidence for different abstract features. In this sense, these interpretations are really inferences that the learners are making about the (described) people on the basis of the evidence provided in the description and other knowledge they bring to bear. That is, the participants are making inferences that the observable features indicate the presence of less observable and more abstract features (e.g., inferring that rides a bike to work indicates athletic). Generally, such inferences to abstract features may make categories cohere, even when the observable features are somewhat more variable. Thus, such interpretations or inferences may be critical in allowing us to ignore differences between category members at the level of observable features, if those observable features are linked to an important, but more abstract and less observable, commonality.

We should point out that with the present materials, it was entirely possible for people to make both interpretations. For example, someone who rides a bike to work could easily be considered both athletic and environmentally aware. Thus, the participants did not have to interpret the observable features in the present experiments, because the features that we used could be linked to both abstract features or could be understood by themselves without their being linked to any abstract feature. Nevertheless, interpretation did indeed occur for our features, and the interpretation had important consequences for what the participants thought about the category as a whole. This suggests an interesting negative aspect of feature interpretation: Once an abstract feature is activated, it may be hard to overcome that feature's influence, even if there are other abstract features that fit equally well with the later presented information. Such an effect would be consistent with many kinds of psychological findings, such as the persistence of first impressions, confirmation biases, or the idea of self-fulfilling prophecies. Overall, the present experiments suggest that feature interpretation is a very common aspect of learning about categories, much more common than might have been expected from the previous research.

Interpretation and Inference. As was made clear in Experiment 1, one need not have special "uninterpreted" features to serve as standards against which other features can be interpreted. Instead, features that share a possible interpretation as an example of a more abstract feature seem to be mutually reinforcing. That is, a particular feature can be interpreted as consistent with a possible interpretation common to features of other highly available category members. An important possibility that has not yet been investigated concerns the degree to which all features may be "interpretable," at least to some degree. For example, it could be that if one learned that a number of people from a particular group were interested in environmental issues, a new person in that group who had political aspirations might be construed as wishing to use a political position to affect some environmentally friendly changes, even though no information was available to directly suggest whether the new person had any environmental leaning.

When one considers again the relationship of feature interpretation to the larger question of inference, it becomes clear that the notion of "interpretation" takes on a broader meaning. That is, one of the most important and common uses of concepts is to fill in unavailable information (see, e.g., Anderson, 1991). For example, a concept can be used to fill in information that is not currently available, such as that a new and unfamiliar bird that is now seen simply sitting on the ground is likely to be able to fly. There is nothing in the information presented by the bird itself that allows this information to be filled in; it is the concept that allows one to infer that the bird is likely to be able to fly. On the other hand, if one had recently encountered a very similar bird that could not fly, one might infer that this bird would not fly. Such inference is usually taken to be at the level of given features (Anderson, 1991), but the present work suggests that many inferences may be made at the level of more abstract features. That is, almost any feature can be taken as some evidence for more than one inference, even if those inferences are contradictory. Thus, there may be few (if any) features that are completely "uninterpretable," in the sense that features may generally provide evidence for many inferences at higher levels of abstraction. Again, this suggests that feature interpretation and inference may be much more common in category learning than previously believed.

Generality of Stimuli and Task. Although we have used verbal descriptions of people as stimuli, we believe that most of the present results are likely to be found with the use of other kinds of stimuli. Similar problems in determining the appropriate feature set arise when one encounters unfamiliar objects (see, e.g., Schyns \& Rodet, 1997). Artifacts are likely to lead to the same sorts of problems as well. For example, imagine simply seeing a new artifact. How does one determine the appropriate features? Well, background knowledge about this type of artifact would certainly be useful. For example, it would probably be very helpful to have some information about the user of the artifact, the purpose for which the artifact was designed, and so on. However, in the absence of such information, one might well make use of similar ar- 
tifacts to try to understand the new artifact, in much the same way as we have seen in the present experiments.

Additionally, feature interpretation probably occurs in various forms in a large number of cognitive tasks. For example, in addition to the effects on category learning investigated here, visual perception involves some form of feature interpretation or creation (Goldstone, 1995), and many cases of real-world problem solving are likely to involve substantial amounts of feature interpretation (see, e.g., Allen, Norman, \& Brooks, 1992). It is certainly true that large portions of most discourses require participants to go far beyond the information explicitly presented in the discourse (Grosz, Pollack, \& Sidner, 1989) and that text comprehension also requires such interpretation in encoding the meaning of the text (Ross \& Bradshaw, 1994). It may be that the factors that affect feature interpretation in category learning will be generalizable to other cognitive tasks as well. This connection to other tasks perhaps becomes more obvious when we think about concept learning as including understanding of what makes the concept cohere. The basic idea of connecting observable aspects of the stimulus to less observable, more abstract aspects is likely to be common across many tasks, and thus, the kinds of feature interpretation and inference that we have demonstrated are also likely to be common to many different tasks.

Assimilation and Contrast. It should be acknowledged that to this point, we have considered only one side of feature interpretation, namely assimilation. That is, we have considered only cases in which it is expected that learners will interpret the critical features in a way that is consistent with abstract features suggested by other similar or proximate instances. It is also possible that there would be cases in which a feature would contrast with the abstract features suggested by other instances (see, e.g., Goldstone, 1995). For example, imagine someone who takes very short, slow, easy bike rides around the neighborhood. Now, suppose that this person reminded you of someone who biked 100 miles per day for exercise (perhaps both have an unusual shade of red hair). Does this make it seem more likely that the person taking the easy rides is doing so for exercise? What if the person taking the easy rides reminded you of someone who took brief walks for exercise? It might be that being reminded of the walker is more likely to make you think of the easy rides as exercise than being reminded of the really extreme exercise of the 100-mile-per-day rider. In comparison with the 100 -mile rides, perhaps the slow short rides do not seem like exercise at all. Generally, it may be that a new item can either be contrasted with or assimilated to highly available abstract features.

\section{Conclusion}

In summary, people learning categories sometimes interpret observable features with respect to abstract features suggested by other instances of the categories be- ing learned. Thus, the assumption of given features in most models of categorization is unwarranted. Instead, theories of category learning must also specify how the set of (interpreted) features arises. Such theories would need to include the general possibility that features occur at multiple levels of abstraction and, specifically, the effects of abstract features suggested by highly available instances (and more general knowledge) on feature interpretation. Most generally, it seems likely that feature interpretation and inference occur frequently, probably at many different levels of feature abstractness, and are critically important in concept learning and likely in many other cognitive tasks.

\section{REFERENCES}

Allen, S. W., Norman, G. R., \& Brooks, L. R. (1992). Experimental studies of learning dermatologic diagnosis: The impact of examples. Teaching \& Learning in Medicine, 4, 35-44.

ANDERSON, J. R. (1991). The adaptive nature of human categorization. Psychological Review, 98, 409-429.

Asch, S. E. (1946). Forming impressions of personality. Journal of $A b$ normal \& Social Psychology, 41, 258-290.

ElIo, R., \& ANDERSON, J. R. (1981). The effects of category generalizations and instance similarity on schema abstraction. Journal of Experimental Psychology: Human Learning \& Memory, 7, 397-417.

ElIO, R., \& ANDERSON, J. R. (1984). The effects of information order and learning mode on schema abstraction. Memory \& Cognition, 12 , 20-30.

GoldSTONE, R. L. (1995). Effects of categorization on color perception. Psychological Science, 6, 298-304.

Grosz, B. J., Pollack, M. E., \& Sidner, C. L. (1989). Discourse. In M. I. Posner (Ed.), Foundations of cognitive science (pp. 437-468). Cambridge, MA: MIT Press.

HinTZMAN, D. L. (1986). "Schema abstraction" in a multiple-trace memory model. Psychological Review, 93, 411-428.

HINTZMAN, D. L. (1988). Judgments of frequency and recognition memory in a multiple-trace memory model. Psychological Review, 95, 528-551.

Komatsu, L. K. (1992). Recent views of conceptual structure. Psychological Bulletin, 112, 500-526.

Medin, D. L. (1989). Concepts and conceptual structure. American Psychologist, 44, 1469-1481.

Medin, D. L., \& BetTger, J. G. (1994). Presentation order and recognition of categorically related examples. Psychonomic Bulletin \& Review, 1, 250-254.

Medin, D. L., \& Florian, J. E. (1992). Abstraction and selective coding in exemplar-based models of categorization. In A. Healy, S. Kosslyn, \& R. Shiffrin (Eds.), From learning processes to cognitive processes: Essays in honor of William K. Estes (Vol. 2, pp. 207-234). Hillsdale, NJ: Erlbaum.

Medin, D. L., Goldstone, R. L., \& Gentner, D. (1993). Respects for similarity. Psychological Review, 100, 254-278.

Medin, D. L., \& Ortony, A. (1989). Psychological essentialism. In S. Vosniadou \& A. Ortony (Eds.), Similarity and analogical reasoning (pp. 179-195). New York: Cambridge University Press.

Medin, D. L., \& SChaffer, M. M. (1978). Context theory of classification learning. Psychological Review, 85, 207-238.

MICHALSKI, R. S. (1983). A theory and methodology of inductive learning. In R. S. Michalski, J. G. Carbonell, \& T. M. Mitchell (Eds.), Machine learning: An artificial intelligence approach (pp. 83-134). Palo Alto, CA: Tioga.

MOONEY, R. J. (1993). Integrating theory and data in category learning. In D. L. Medin (Ed.), The psychology of learning and motivation (Vol. 29, pp. 189-218). San Diego: Academic Press. 
Murphy, G. L. (1993). Theories and concept formation. In I. van Mechelen, J. Hampton, R. Michalski, \& P. Theuns (Eds.), Categories and concepts: Theoretical views and inductive data analysis (pp. $173-$ 200). London: Academic Press.

MurPhy, G. L., \& MEDIN, D. L. (1985). The role of theories in conceptual coherence. Psychological Review, 92, 289-316.

MuRPHy, G. L., \& SPalding, T. L. (1995). Knowledge, similarity, and concept formation. Psychologica Belgica, 35, 127-144.

Pevtzow, R., \& Goldstone, R. L. (1994). Categorization and the parsing of objects. In Proceedings of the Sixteenth Annual Conference of the Cognitive Science Society (pp. 717-722). Hillsdale, NJ: Erlbaum.

Posner, M. I., \& Keele, S. W. (1968). On the genesis of abstract ideas Journal of Experimental Psychology, 77, 353-363.

Posner, M. I., \& KeELe, S. W. (1970). Retention of abstract ideas. Journal of Experimental Psychology, 83, 304-308.

Rosch, E. (1975). Cognitive representation of semantic categories Journal of Experimental Psychology: General, 104, 192-233.

Rosch, E. (1978). Principles of categorization. In E. Rosch \& B. B Lloyd (Eds.), Cognition and categorization (pp. 27-48). Hillsdale, NJ: Erlbaum.

Rosch, E., \& MERvis, C. B. (1975). Family resemblance: Studies in the internal structure of categories. Cognitive Psychology, 7, 573-605.

Ross, B. H., \& BRADSHaw, G. L. (1994). Encoding effects of remindings. Memory \& Cognition, 22, 591-605.

Ross, B. H., Perkins, S. J., \& Tenpenny, P. L. (1990). Remindingbased category learning. Cognitive Psychology, 22, 460-492.

Schyns, P. G., Goldstone, R. L., \& ThiBaut, J.-P. (1998). The development of features in object concepts. Behavioral \& Brain Sciences, 21, $1-17$

SCHYNS, P. G., \& MURPHY, G. L. (1994). The ontogeny of part representation in object concepts. In D. L. Medin (Ed.), The psychology of learning and motivation (Vol. 31, pp. 305-349). San Diego: Academic Press.

SCHYNS, P. G., \& RodeT, L. (1997). Categorization creates functional features. Journal of Experimental Psychology: Learning, Memory, \& Cognition, 23, 681-696.

Smith, E. E., \& Medin, D. L. (1981). Categories and concepts. Cambridge, MA: Harvard University Press.

SPALDING, T. L., \& FoRD, M. A. (1998). Background knowledge and memory for category features. Manuscript submitted for publication.

SPALDING, T. L., \& MURPHY, G. L. (1999). What is learned in knowledgerelated categories? Evidence from typicality and feature frequency judgments. Memory \& Cognition, 27, 856-867.

SPalding, T. L., \& Phillips, V. L. (1998). What happens when features mismatch background knowledge about a category? Evidence from typicality, categorization, and recognition judgments. Manuscript submitted for publication.

Spalding, T. L., \& Ross, B. H. (1994). Comparison-based learning: Effects of comparing instances during category learning. Journal of Experimental Psychology: Learning, Memory, \& Cognition, 20, 12511263.

Thibaut, J.-P., \& Schyns, P. G. (1995). The development of feature spaces for similarity and categorization. Psychologica Belgica, 35, 167-185.

WISNIEWSKI, E. (1995). Prior knowledge and functionally relevant features in concept learning. Journal of Experimental Psychology: Learning, Memory, \& Cognition, 21, 449-468.

WISNIEWSKI, E., \& MEDIN, D. L. (1991). Harpoons and long sticks: The interaction of theory and similarity in rule induction. In D. H. Fisher, M. J. Pazzani, \& P. Langley (Eds.), Concept formation: Knowledge and experience in unsupervised learning (pp. 237-278). San Mateo, CA: Morgan Kaufman.

WisNiEwski, E., \& MEdin, D. L. (1994). On the interaction of theory and data in concept learning. Cognitive Science, 18, 221-281.

(Manuscript received March 17, 1998;

revision accepted for publication April 7, 1999.) 